\title{
Assessment of Regional Myocardial Function via Statistical Features in MR Images
}

\author{
Mariam Afshin ${ }^{1,3}$, Ismail Ben Ayed ${ }^{2}$, Kumaradevan Punithakumar ${ }^{2}$, \\ Max W.K. Law ${ }^{1,2}$, Ali Islam ${ }^{4}$, Aashish Goela ${ }^{5}$, Ian Ross ${ }^{5}$, \\ Terry Peters ${ }^{1,3}$, and Shuo $\mathrm{Li}^{1,2}$ \\ 1 University of Western Ontario, London, ON, Canada \\ 2 GE Healthcare, London, ON, Canada \\ 3 Robarts Research Institute, London, ON, Canada \\ 4 St. Joseph's Health Care, London, ON, Canada \\ ${ }^{5}$ London Health Science Center, London, Ontario, Canada
}

\begin{abstract}
Early and accurate detection of Left Ventricle (LV) regional wall motion abnormalities significantly helps in the diagnosis and followup of cardiovascular diseases. We present a regional myocardial abnormality detection framework based on image statistics. The proposed framework requires a minimal user interaction, only to specify initial delineation and anatomical landmarks on the first frame. Then, approximations of regional myocardial segments in subsequent frames were systematically obtained by superimposing the initial delineation on the rest of the frames. The proposed method exploits the Bhattacharyya coefficient to measure the similarity between the image distribution within each segment approximation and the distribution of the corresponding user-provided segment. Linear Discriminate Analysis (LDA) is applied to find the optimal direction along which the projected features are the most descriptive. Then a Linear Support Vector Machine (SVM) classifier is employed for each of the regional myocardial segments to automatically detect abnormally contracting regions of the myocardium. Based on a clinical dataset of 30 subjects, the evaluation demonstrates that the proposed method can be used as a promising diagnostic support tool to assist clinicians.
\end{abstract}

\section{Introduction}

Heart failure is a prevalent disease that can be caused by various heart conditions, in particular, ischemic heart disease (IHD) [1. The decrease of blood supply produced by coronary artery stenosis impairs the contractile properties of specific myocardial areas. This deviates the normal regional wall motion and contractility patterns of the myocardium, especially the left ventricle (LV). Early and accurate detection of LV regional wall motion abnormalities significantly helps in the diagnosis and follow-up of IHD 2. In routine clinical use, cardiac function is estimated by visual assessment and interpretation of LV and, therefore, it is highly subject-dependent. Alternatively, computer-aided detection

G. Fichtinger, A. Martel, and T. Peters (Eds.): MICCAI 2011, Part III, LNCS 6893, pp. 107-114, 2011. (C) Springer-Verlag Berlin Heidelberg 2011 
systems have been attempted in recent years in order to automatically analyze LV myocardial function quantitatively [3], and to classify hearts into normal or abnormal groups [4. In clinical practice, the regional myocardial function is commonly scored by following American Heart Association (AHA) standards [5], where the LV is divided into 17 segments. Existing regional heart function analysis methods are based on information theoretic measures and unscented Kalman filter approaches [6], differentiable manifolds [7, independent component analysis classifier [8], pattern recognition technique based on intra-segment correlation [9], and tensor-based classification [10]. Most of the existing methods require extensive user interaction or computationally expensive segmentation algorithms. This study investigates assessment of regional myocardial function using MR statistics and starting from a minimal user input. Typically cardiac MR data consist of 10 sequences, each comprising 20 or 25 temporal image frames. From a simple user input, we computed image statistics that are related to myocardium function. Given a user-provided delineation of the first frame, approximations of regional myocardial segments in subsequent frames were systematically obtained by superimposing the initial delineation on the rest of the frames. The proposed method exploits the Bhattacharyya coefficient [1] to measure the similarity between the image distribution within each segment approximation and the distribution of the corresponding user-provided segment. Linear Discriminate Analysis (LDA) is applied to find the optimal direction along which the projected features are the most descriptive. Linear Support Vector Machine (SVM) classifier is then employed for each of the regional myocardial segments to automatically detect abnormal functional regions of the myocardium. The proposed method performs significantly better than other recent methods and requires fewer computational resources. The evaluations performed on a clinical dataset of 30 subjects show that the proposed method is a promising diagnostic support tool.

\section{Constructing Image Statistics}

We consider image statistics as representative features to classify regional myocardium into normal or abnormal classes. Let $\mathcal{I}$ be a set of cardiac MR images of a single slice containing $N$ frames 1 . Let $\mathbf{I}$ be a reference image which is an end-diastolic frame corresponding to the largest volume during cardiac cycle, whose delineation is given as depicted in Fig. 1(a). Let $\Gamma_{\text {in }}, \Gamma_{\text {out }}:[0,1] \rightarrow \Omega$ denote respectively the corresponding manual endo and epi-cardial boundaries of I. We divide I into $M$ regional segment 2 following the AHA standard [5], and using anatomical landmarks 3 . Fig. 1(b) shows the regional segments for I. For $(n, m) \in[1 \ldots N] \times[1 \ldots M]$, let $I_{n m}$ denotes the regional cardiac segment corresponding to segment $m$ in frame $n$, and $\Gamma_{\text {out }}^{n m}$ the boundary of $I_{n m}$ (refer

${ }^{1}$ The number of frames $N$ is typically equal to 20 or 25 .

${ }^{2} M$ would be 4, 6, and 6 for apical, mid-cavity and basal slices, respectively.

${ }^{3}$ As suggested by [5] the attachment of the right ventricular wall to the LV is used to identify and separate the septum from the LV anterior and inferior free walls. 


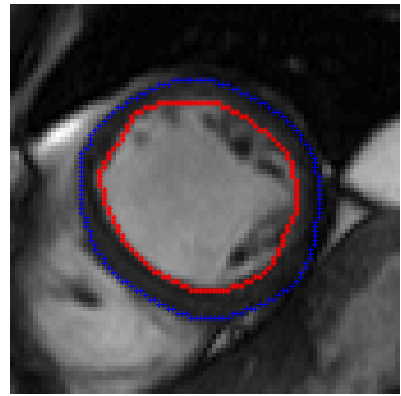

(a)

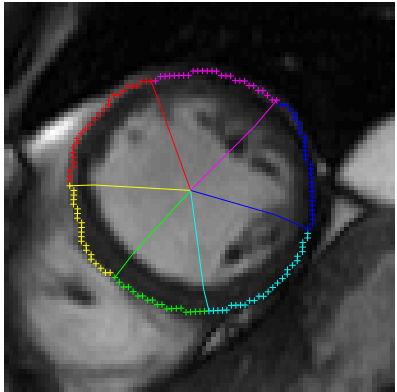

(b)

Fig. 1. (a) Manual delineation of the reference image I for a mid-cavity slice. (b) Regional segments of the reference image $\mathbf{I}$ for mid-cavity slice.

to Fig 2 (a)). The classification procedures are identical for apical, mid-cavity and basal slices. Let us now superimpose the region defined by the epi-cardial boundary $\Gamma_{\text {out }}^{n m}$ to the other frames in the sequence as shown in Fig. 2(a-c), and compute the corresponding image statistics (Fig. 2 (d-f)). We define $\mathbf{R}_{\boldsymbol{\Gamma}} \subset \Omega$ to be the region enclosed within $\Gamma \in\left\{\Gamma_{\text {in }}, \Gamma_{\text {out }}, \Gamma_{\text {out }}^{n m}\right\}$, and $P_{\mathbf{R}_{\Gamma}, \text { I }}$ the intensity distribution of I within region $\mathbf{R}_{\Gamma}$ :

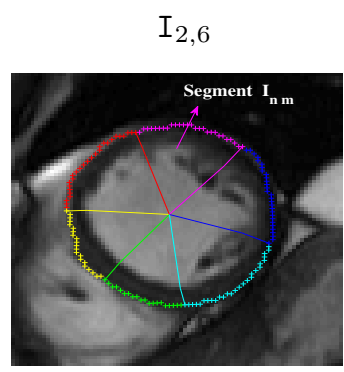

(a)

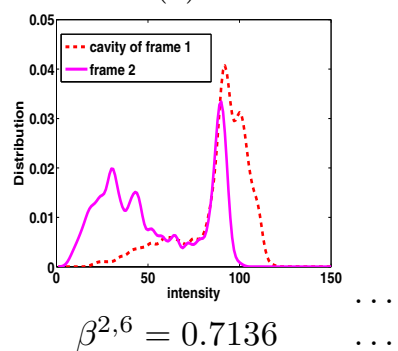

(d)

...
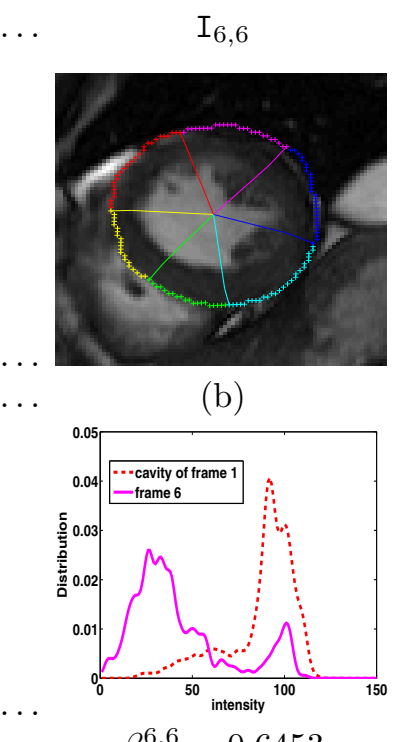

(e)
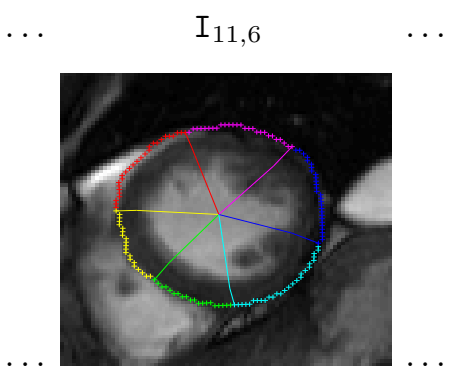

(c)

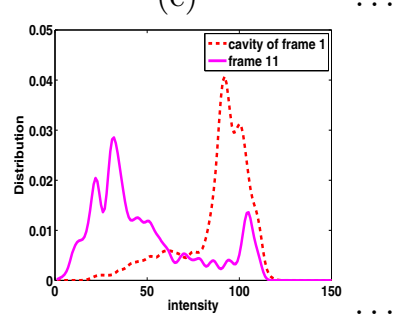

$\ldots \quad \beta^{11,6}=0.6947 \quad \ldots$

(f)

Fig. 2. (a-c): Regional myocardial segments superimposed on subsequent frames. (d-f): the corresponding image statistics. 


$$
P_{\mathbf{R}_{\Gamma}, \mathrm{I}}(z)=\frac{\int_{\mathbf{R}_{\Gamma}} K(z-\mathrm{I}) d x}{a_{\mathbf{R}_{\Gamma}}}, \quad K(y)=\frac{1}{\sqrt{2 \pi \sigma^{2}}} \exp ^{-\frac{y^{2}}{2 \sigma^{2}}},
$$

where $a_{\mathbf{R}_{\Gamma}}$ is the area inside region $\mathbf{R}_{\Gamma}$ and $K$ is the Gaussian kernel [12. Now let $P_{\mathbf{R}_{\Gamma_{i n}}, \mathbf{I}}$ denotes the image distribution corresponding to the blood within the cavity of the reference image $\mathbf{I}$ whose delineation is given by the user (refer to the distributions depicted by the discontinuous, red curves in Fig. 2 (d-f)). Furthermore, we estimate the distribution inside $\Gamma_{\text {out }}^{n m}$ by $P_{\mathbf{R}_{\Gamma_{\text {out }}^{n m} \text {, I }}}$ in all other frames in the sequence as shown in Fig. 2(d-f) (continuous, pink curves). Now we consider the similarity measurement, $\beta$, between image distribution $P_{\mathbf{R}_{\Gamma_{i n}}, \mathbf{I}}$ corresponding to the blood in the cavity of the reference image $\mathbf{I}$ and distribution $P_{\mathbf{R}_{\Gamma_{\text {out }}^{n m}, \mathrm{I}}^{\text {I }}}$ :

$$
\beta^{n, m}=\mathbf{B}\left(P_{\mathbf{R}_{\Gamma_{i n}}, \mathbf{I}}, P_{\mathbf{R}_{\Gamma_{\text {out }}^{n m}, \mathrm{I}}^{n m}}\right), \quad \mathbf{B}(f, g)=\int_{R^{+}} \sqrt{f g} d z
$$

$\mathbf{B}(f, g)$ is the Bhattacharyya coefficient measuring the overlap (similarity) between distributions $f$ and $g$. The range of the Bhattacharyya coefficient is $[0,1]$, with 0 indicating no overlap between the distributions and 1 indicating a perfect match. The fixed $[0,1]$ range of the Bhattacharyya coefficient affords a conveniently practical appraisal of the similarity. We expect that measurement $\beta^{n, m}$ is related to the amount of blood in the corresponding segment $I_{n m}$, a relationship that is demonstrated experimentally by the typical example in Fig. 2, Such similarity is reasonable because the more overlap between the image distribution within cavity and the distribution within regional segment $I_{n m}$, the higher the blood volume inside regional segment $I_{n m}$. When a regional myocardial muscle does not contract properly, the distribution of blood within $\mathbf{R}_{\Gamma_{\text {out }}^{n m}}$, I, does not change and, therefore, $\beta^{n, m} \forall i \in\{1, \ldots, 20\}$, can be used as a criterion to assess the myocardial function of segment $I_{n m}$. We then employ a linear SVM classifier and use the estimated $\beta^{n, m}$ (there are $20 \beta^{n, m}$ for one regional segment) as features to classify regional myocardial segments as normal or abnormal.

\section{LDA and Linear SVM Classifier for Regional Myocardial Abnormality Detection}

We applied Linear Discriminant Analysis (LDA) to reduce the dimensionality of feature vectors, $\boldsymbol{\beta}^{m}=\left\{\beta^{n, m}\right\}$ s.t. $n \in\{1, \ldots, 20\}$, while maximizing the distance between normal and abnormal classes. This can be achieved by projecting estimated feature vectors, $\boldsymbol{\beta}^{m}$, to a new lower-dimensional feature space of $\beta_{p}{ }^{m}$ s.t. $\beta_{p}{ }^{m}=F_{L D A}\left(\boldsymbol{\beta}^{m}\right) . F_{L D A}$ transforms $\boldsymbol{\beta}^{m}$ to $\beta_{p}{ }^{m}$ to discriminate among abnormal and normal classes [13. Subsequently, a linear Support Vector Machine (SVM) classifier is used to identify the decision boundary to classify the regional myocardial segments into normal and abnormal categories. The vectors near the decision boundary are called support vectors. We used linear SVM classifier to maximize the margin between the support vectors of both classes. We trained the linear SVM classifier by providing $\beta_{p}{ }^{m}$ and the associated labels of normal or abnormal obtained from ground truth by an expert radiologist. 


\section{Experiments}

Data acquisition. A set of $2 \mathrm{D}$ short-axis cine magnetic resonance (MR) images of 30 subjects were acquired over the cardiac cycle on a $1.5 \mathrm{~T}$ scanner with fastimaging employing a steady-state acquisition (FIESTA) image sequence. The acquisition parameters were: $\mathrm{TR}=2.98 \mathrm{~ms}, \mathrm{TE}=1.2 \mathrm{~ms}$, flip angle=30 degree, and slice thickness $=10 \mathrm{~mm}$. The data contain 90 short-axis image sequences, each consisting of 20 functional 2D images. The results for 480 myocardial segments from apical, mid-cavity and apical were compared with ground truth classifications by an expert radiologist 4 .

Applying Linear Discriminant Analysis. After estimating image features, we applied a LDA transformation for each of regional myocardial segments individually. Fig. 3 shows the projected features $\beta_{p}{ }^{m}$ after applying LDA transformation for regional segment 3 of apical, mid-cavity and basal slices. The results show that projected features for the apical cases are more discriminative than basal and mid-cavity regions. This can be explained by the fact that there are no papillary muscles in apical slices and, therefore, estimation of the distribution corresponding to blood within cavity of apical slice is less challenging compared to basal and mid-cavity slices.

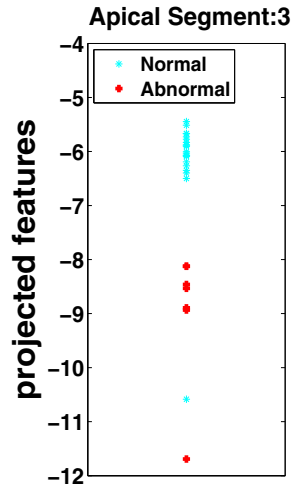

(a)

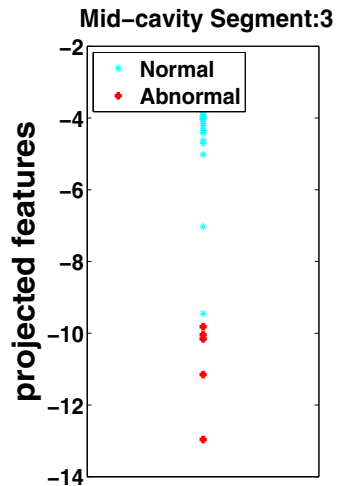

(b)

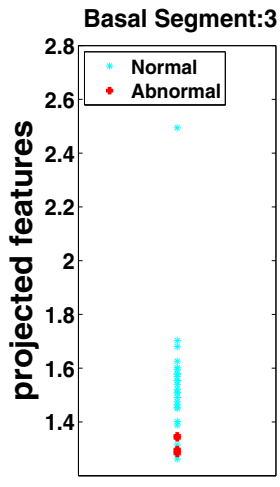

(c)

Fig. 3. projected features, $\beta_{p}^{m}$, after applying LDA transformation for regional segment 3 of apical, mid-cavity and basal slices

Linear SVM Classifier. We used 16 linear SVM classifiers to assess the 16 regional myocardial segments (normal/abnormal). Fig. 4 shows that the decision boundary separates the normal and abnormal classes using linear SVM. The decision boundary for apical is more reliable than the corresponding regional segment in the basal slice. The greater the distance between the support vectors

\footnotetext{
${ }^{4}$ Among the 480 myocardial segments, 389 segments were marked as normal and 91
} as abnormal. 


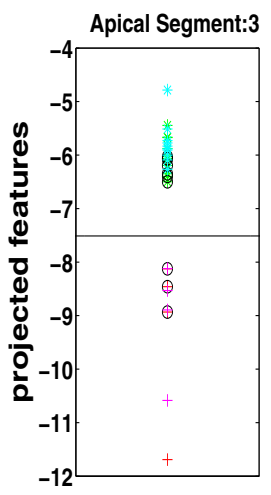

(a)

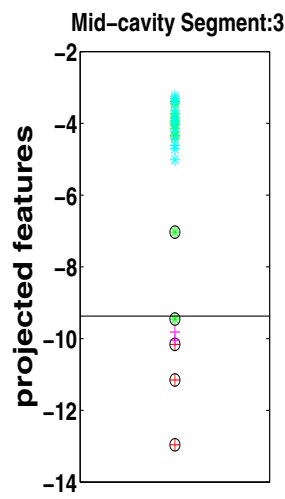

(b)

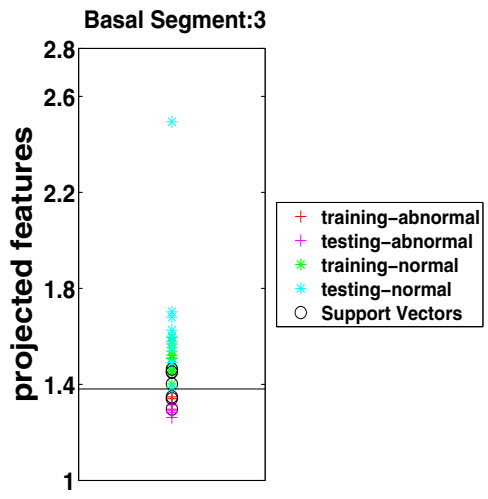

(c)

Fig. 4. Decision boundary and support vectors for regional segment 3 of apical, midcavity and basal slices

of normal and abnormal classes, the more reliable the decision boundary. The decision boundary in the case of mid-cavity slices suffers from misclassification because of the papillary muscles that are connected to myocardial wall.

Classification performance. We used two criteria to measure the performance of each classifier, namely the ROC, Receiver Operating Characteristics, curves with corresponding AUCs, Area Under the ROC Curve, and the Bhattacharyya measure [1] to assess the discriminative power of the features. Furthermore, we assessed the performance of the proposed approach by training our algorithm using $2 / 3$ of the dataset and testing on the rest of the data.

ROC, AUC and Bhattacharyya measure. We show the ROC curves for classifier elements in Fig. 5. The figures show that the proposed method based on the Bhattacharyya coefficient is a reliable approach, for detecting regional abnormality in cardiac MR images. Figs. [5 (a), (b) and (c) show that apical segments are better classified than basal while basal slices are better classified than

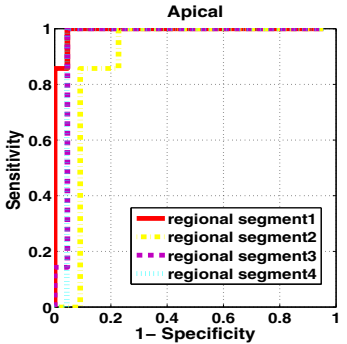

(a) Apical

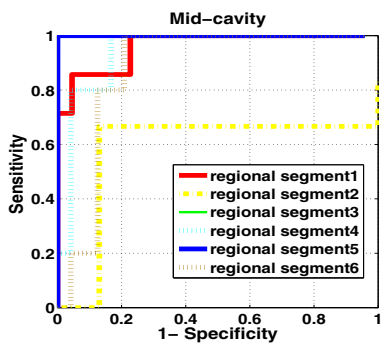

(b) Mid-cavity

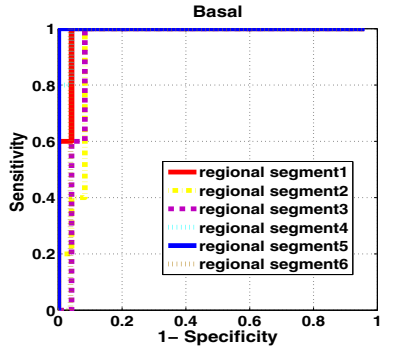

(c) Basal

Fig. 5. Receiver operating characteristics of classifiers. The closer the curve to the left hand top corner, the better the classification performance. 
Table 1. The area under the curve corresponding to Fig. [5 and the Bhattacharyya distance metric $(\mathcal{B})$ of normal/abnormal distributions. The higher the values, the more discriminative the ability of the classifier.

\begin{tabular}{lcc}
\hline & AUC & $\begin{array}{c}\text { Bhattacharyya distance } \\
\text { metric }(\mathcal{B})\end{array}$ \\
\hline Apical & 0.94 & 0.91 \\
Mid-cavity & 0.85 & 0.85 \\
Basal & 0.87 & 0.94 \\
\hline
\end{tabular}

mid-cavity slices. The AUCs corresponding to ROC curves in Fig. [5] are reported in Table 1. We also used the Bhattacharyya distance metric, $\mathcal{B}$, to evaluate the overlap between the distribution of features over normal and abnormal classes. The higher the $\mathcal{B}$, the more discriminative the classifier. The $\mathcal{B} \mathrm{s}$ in Table 1 are consistent with ROC/AUC evaluations.

Table 2. The classification accuracy computed by leaving-one-third-of-the-subjectsout. The proposed method achieved an overall classification accuracy of $91.54 \%$.

\begin{tabular}{lccc}
\hline & Sensitivity (\%) & Specificity (\%) & Accuracy (\%) \\
\hline Apex & 100.0 & 90.91 & 92.86 \\
Mid-cavity & 93.33 & 92.93 & 90.48 \\
Base & 83.0 & 94.45 & 91.3 \\
\hline
\end{tabular}

We also evaluated the performance of the classifier by computing the accuracy, specificity and sensitivity over datasets. Table 2 reports the results. The overall classification accuracy is equal to $91.5 \%$, with a sensitivity of $92.1 \%$ and specificity of $92.8 \%$. The highest performance was achieved for apical slices with average of $92.9 \%$ for accuracy, $100 \%$ for sensitivity, and $90.9 \%$ for specificity.

\section{Conclusions}

We presented a regional cardiac abnormality detection method based on the statistics of the image, which were estimated based on user-provided delineation of the first frame. Then, from this simple input, we estimated image statistics for each regional segment, and used them as features for regional heart abnormality classification. The LDA was applied to estimate projected features and a linear SVM classifier was used to classify regional LV segments into normal or abnormal classes. The experimental analysis was carried out over $90 \times 20$ segmented LV cavities of short-axis MR images obtained from 30 subjects, and demonstrated that the proposed method performs significantly better than other state-of-art methods, and can lead to a promising diagnostic support tool to assists clinicians. 


\section{References}

1. Bleumink, G.S., Knetsch, A.M., Sturkenboom, M.C., Straus, S.M., Hofman, A., Deckers, J.W., Witteman, J.C., Stricker, B.H.: Quantifying the heart failure epidemic: Prevalence, incidence rate, lifetime risk and prognosis of heart failure - the rotterdam study. European Heart Journal 25, 1614-1619 (2004)

2. Buckberg, G.: Left ventricular form and function: Scientific priorities and strategic planning for development of new views of disease. Circulation 110, e333-e336 (2004)

3. Sundar, H., Davatzikos, C., Biros, G.: Biomechanically-constrained 4D estimation of myocardial motion. In: Yang, G.-Z., Hawkes, D., Rueckert, D., Noble, A., Taylor, C. (eds.) MICCAI 2009. LNCS, vol. 5762, pp. 257-265. Springer, Heidelberg (2009)

4. Punithakumar, K., Li, S., Ayed, I.B., Ross, I., Islam, A., Chong, J.: Heart motion abnormality detection via an information measure and bayesian filtering. In: Yang, G.-Z., Hawkes, D., Rueckert, D., Noble, A., Taylor, C. (eds.) MICCAI 2009. LNCS, vol. 5762, pp. 373-380. Springer, Heidelberg (2009)

5. Cerqueira, M.D., Weissman, N.J., Dilsizian, V., Jacobs, A.K., Kaul, S., Laskey, W.K., Pennell, D.J., Rumberger, J.A., Ryan, T., Verani, M.: Standardized myocardial segmentation and nomenclature for tomographic imaging of the heart: A statement for healthcare professionals from the cardiac imaging committee of the council on clinical cardiologyof the American Heart Association. Circulation 105(4), 539-542 (2002)

6. Punithakumar, K., Ayed, I.B., Islam, A., Ross, I.G., Li, S.: Regional heart motion abnormality detection via information measures and unscented kalman filtering. In: Jiang, T., Navab, N., Pluim, J.P.W., Viergever, M.A. (eds.) MICCAI 2010. LNCS, vol. 6361, pp. 409-417. Springer, Heidelberg (2010)

7. Garcia-Barnes, J., Gil, D., Badiella, L., Hernàndez-Sabaté, A., Carreras, F., Pujadas, S., Martí, E.: A normalized framework for the design of feature spaces assessing the left ventricular function. IEEE Transaction on Medical Imaging 29(3), 733-745 (2010)

8. Suinesiaputra, A., Frangi, A., Kaandorp, T., Lamb, H., Bax, J., Reiber, J., Lelieveldt, B.: Automated detection of regional wall motion abnormalities based on a statistical model applied to multislice short-axis cardiac MR images. IEEE Transaction on Medical Imaging 28(4), 595-607 (2009)

9. Lu, Y., Radau, P., Connelly, K., Dick, A., Wright, G.: Pattern recognition of abnormal left ventricle wall motion in cardiac MR. In: Yang, G.-Z., Hawkes, D., Rueckert, D., Noble, A., Taylor, C. (eds.) MICCAI 2009. LNCS, vol. 5762, pp. 750-758. Springer, Heidelberg (2009)

10. Mansor, S., Noble, J.: Local wall motion classification of stress echocardiography using a hidden Markov model approach. In: The IEEE International Symposium in Biomedical Imaging: From Nano to Macro, pp. 1295-1298 (2008)

11. Ben Ayed, I., Ross, I., Li, S.: Embedding overlap priors in variational left ventricle tracking. IEEE Transaction on Medical Imaging 28(12), 1902-1913 (2009)

12. Ben Ayed, I., Li, S., Ross, I.: A statistical overlap prior for variational image segmentation. International Journal of Computer Vision 85(1), 115-132 (2009)

13. Kim, H.C., Kim, D., Bang, S.Y.: Face recognition using lda mixture model. Pattern Recogn. Lett. 24, 2815-2821 (2003) 